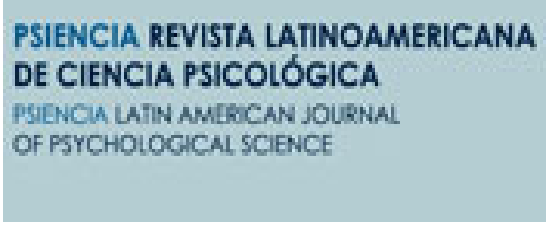

PSIENCIA REVISTA LATINOAMERICANA

DE CIENCIA PSICOLOCICA OF PSYCHOLOCICAL SCIENCE

PSIENCIA. Revista Latinoamericana de Ciencia Psicológica

ISSN: 2250-5490

contacto@psiencia.org

Asociación para el Avance de la Ciencia

Psicológica

Argentina

Rivera Varela, Bertha Leticia; García Lirios, Cruz; García Estrada, Erle; Rosas Ferrusca, Francisco Javier; Limón Domínguez, Gerardo Arturo; Carreón Guillén, Javier; Hernández Valdés, Jorge; Morales Flores, María de Lourdes CONTRASTE DE UN MODELO DE DESCONFIANZA POLÍTICA

PSIENCIA. Revista Latinoamericana de Ciencia Psicológica, vol. 7, núm. 1, 2015, pp. 1-8 Asociación para el Avance de la Ciencia Psicológica

Buenos Aires, Argentina

Disponible en: http://www.redalyc.org/articulo.oa?id=333139283001

Cómo citar el artículo

- Número completo

- Más información del artículo

- Página de la revista en redalyc.org 


\section{CONTRASTE DE UN MODELO DE DESCONFIANZA POLÍTICA}

\section{CONTRAST OF A MODEL OF MISTRUST TOWARDS POLITICS}

Bertha Leticia Rivera Varela1 ${ }^{1}$ Cruz García Lirios ${ }^{2}$, Erle García Estrada ${ }^{1,3}$, Francisco Javier Rosas Ferrusca', Gerardo Arturo Limón Domínguez ${ }^{4}$, Javier Carreón Guillén', Jorge Hernández Valdés ${ }^{1,5}$, María de Lourdes Morales Flores ${ }^{1}$

${ }^{1}$ Universidad Nacional Autónoma de México, ${ }^{2}$ Instituto Politécnico Nacional, ${ }^{3}$ Centro Universitario de España y México, ${ }^{4}$ Universidad Pedagógica Nacional de México, ${ }^{5}$ Colegio de Estudios de Posgrado de la Ciudad de México

briveravarela@uaemex.mx

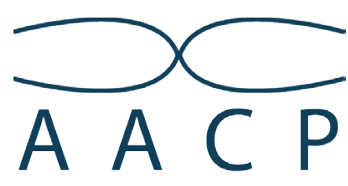

ASOCIACIÓN PARA EL AVANCE DE LA CIENCIA PSICOLÓGICA

doi: $10.5872 / p s i e n c i a / 7.1 .21$ (c) $2015 \cdot$ www.psiencia.org

Cómo citar este artículo:

Rivera Varela, B. L., García Lirios, C., García Estrada, E., Rosas Ferrusca, F. J., Limón Domínguez, G. A., Carreón Guillén, J., ...Morales Flores, M. L. (2015). Contraste de un modelo de desconfianza política. PSIENCIA. Revista Latinoamericana de Ciencia Psicológica, 7, 1-8

Resumen: En virtud de que los estudios de las emociones relativas a la inseguridad han establecido asociaciones directas, positivas y significativas con la evaluación de políticas de prevención del delito o combate a la delincuencia, el objetivo del presente estudio fue establecer las relaciones entre factores emocionales y evaluaciones de la procuración de justicia. Para tal propósito se utilizó una muestra no probabilística de 170 habitantes de una comunidad. Se estimaron la confiabilidad y la validez de cinco inventarios que midieron ansiedad, preocupación, enojo y miedo a eventos cotidianos de inseguridad así como desconfianza a las autoridades. A partir de un modelo estructural y parámetros de dependencia, ajuste y residuales $\left(X^{2}\right.$ $=1.0808 ; 3 \mathrm{gl} ; p=.613 ; \mathrm{GFI}=.984 ; \mathrm{AGFI}=.921 ; \mathrm{NFI}=.867 ; R M R=.058 ; R M S E A=.090)$, se aceptaron nueve de once hipótesis de las cuales sólo la relación entre ansiedad y enojo así como miedo y desconfianza fueron consideradas espurias, aunque la preocupación determinó a la desconfianza $(\beta=.26)$. Los resultados fueron discutidos con los hallazgos reportados por la literatura.

Palabras clave: Delincuencia - Seguridad - Emociones - Preocupación - Confianza

Abstract: Given that the study of emotions concerning insecurity have established direct, significant and positive correlations in the evaluation of policies for the prevention or fighting against crime, the objective of this study was to establish the relationship between emotional factors and assessments of law enforcement. For this purpose we used a nonrandom sample of 170 residents of a community. We estimated the reliability and validity of five inventories that measured anxiety, worry, anger and fear of of everyday events of insecrutity and distrust to authorities. From a structural model and parameter dependence and residual fit $\left(X^{2}=\right.$ $1.0808,3 \mathrm{df}, P=.613, \mathrm{GFI}=.984, \mathrm{AGFI}=.921, \mathrm{NFI}=.867, R M R=.058, R M S E A=.090)$, nine of eleven were accepted hypothesis of which only the relationship between anxiety and anger and fear and distrust were considered spurious, although concern determined mistrust $(\beta=.26)$. The results were discussed with the findings reported in the literature.

Keywords: Crime - Safety - Emotions - Caring - Trust 


\section{INTRODUCCIÓN}

En el marco de la seguridad pública, la relación entre los ciudadanos y las autoridades locales puede ser analizada a partir de sus emociones (tabla 1). La opinión pública, en tanto evaluadora de las políticas públicas, está conformada más por sentimientos que razonamientos en torno al sistema judicial. En este sentido, la clase política en referencia a la esfera civil, es evaluada por afectos que se derivan de su acción gubernamental en materia de prevención del delito o combate a la delincuencia organizada.

Las emociones, a diferencia de los razonamientos, son procesos automáticos, improvisados y heurísticos que activan experiencias con perso- nas $u$ objetos en situaciones de riesgo o incertidumbre.

En el caso de la información difundida por los medios de comunicación, las emociones incrementan o disminuyen el contenido de un mensaje. Es decir, la ciudadanía que desea informarse sobre un acontecimiento específico está expuesta a procesar emocionalmente la información circundante, empero una vez que el evento ha ocurrido, la persuasión se disipa poco a poco. El ciclo reinicia cuando otro evento es esperado como una amenaza que por su lejanía sólo generará preocupación, aunque su cercanía implica una acción concreta para afrontar el problema.

Los estudios psicológicos de las emociones, en materia de seguridad pública, han identificado

Tabla 1. Estado del arte

\begin{tabular}{|c|c|c|c|c|}
\hline Año & Autor & Muestra & Instrumentos & Hallazgos \\
\hline 2010 & $\begin{array}{l}\text { Bolivar, } \\
\text { Contreras, } \\
\text { Jiménez \& } \\
\text { Chaux }\end{array}$ & $\begin{array}{l}\text { Estudiantes } \\
(N=227)\end{array}$ & $\begin{array}{l}\text { Escala de Actitu- } \\
\text { des hacia el Robo }\end{array}$ & $\begin{array}{l}\text { Establecieron diferencias significativas entre hombres y mujeres }(F= \\
8.09 ; p=.005) \text {, así como entre entrevistas y grupos focales }(F=49.61 \\
p=.000) \text { respecto al robo }\end{array}$ \\
\hline 2010 & Elizalde & $\begin{array}{l}\text { Profesores } \\
(N=130)\end{array}$ & $\begin{array}{l}\text { Modos de Afronta- } \\
\text { miento de Estrés }\end{array}$ & $\begin{array}{l}\text { Estableció diferencias significativas entre edad y sexo respecto a estilos } \\
\text { de afrontamiento de la violencia; apoyo social, autocontrol, confronta- } \\
\text { ción, responsabilidad, distanciamiento y evitación }\end{array}$ \\
\hline 2010 & $\begin{array}{l}\text { Fernández, } \\
\text { Revilla \& } \\
\text { Domínguez }\end{array}$ & $\begin{array}{l}\text { Estudiantes } \\
(N=8)\end{array}$ & $\begin{array}{l}\text { Análisis de Con- } \\
\text { tenido }\end{array}$ & $\begin{array}{l}\text { Las emociones (nerviosismo, ansiedad, disgusto, repugnancia, horror, } \\
\text { enojo, miedo, catarsis e insensibilidad) inmediatas y diferidas de los } \\
\text { contenidos de la televisión propician narrativas de responsabilidad y } \\
\text { culpa. Son construcciones derivadas de discursos mediáticos en los que } \\
\text { se enlazan valores, actitudes y acciones. La primera reacción ante la } \\
\text { inseguridad y la violencia es emocional }\end{array}$ \\
\hline 2010 & $\begin{array}{l}\text { Gibson, Sul- } \\
\text { livan, Jones } \\
\text { \& Piquero }\end{array}$ & $\begin{array}{l}\text { Residentes } \\
(N=8772)\end{array}$ & $\begin{array}{l}\text { Self-Control } \\
\text { Temperament } \\
\text { Instrument }\end{array}$ & $\begin{array}{l}\text { Encontraron efectos significativos del contexto del barrio sobre el auto- } \\
\text { control, aunque las características individuales en interrelación con las } \\
\text { características del barrio diluyeron la varianza explicada. En el caso de } \\
\text { los estilos parentales de supervisión }(r=.07) \text {, entusiasta }(r=-.08) \text { y } \\
\text { hostil }(r=-.10) \text { correlacionaron significativamente con el autocontrol }\end{array}$ \\
\hline 2010 & Malone & Estudiantes & $\begin{array}{l}\text { Latin American } \\
\text { Public Opinion } \\
\text { Project }\end{array}$ & $\begin{array}{l}\text { En ciudades con una alta tasa de criminalidad, la confianza en el sis- } \\
\text { tema de justicia está determinada por el temor a la delincuencia local ( } \beta \\
=-.146 ; p=.000) \text {, la evaluación de la acción institucional }(\beta=.737 ; p= \\
.000) \text {, la edad }(\beta=-.0004 ; p=.05) \text {, el tamaño del municipio }(\beta=-.052 ; p \\
=.05) \text {. La confianza hacia la policía es influida por la victimización per- } \\
\text { sonal }(\beta=-.175 ; p=.000) \text {, la delincuencia local }(\beta=-.154 ; p=.0000) \text {, } \\
\text { la evaluación de la acción gubernamental }(\beta=.437 ; p=.001) \text {. La con- } \\
\text { fianza en los derechos humanos esta incidida por la delincuencia local } \\
\text {-.091; } p=.0000) \text {, la evaluación institucional }(\beta=.558 ; p=.000) \text {, el sexo } \\
\text { masculino }(\beta=-.158 ; p=.000) \text { y el tamaño del municipio }(\beta=-.046 ; p \\
=.05) \text {. En el caso de las ciudades con un bajo índice delictivo, destaca } \\
\text { la evaluación de las instituciones como predictor de la confianza en el } \\
\text { sistema de justicia }(\beta=.585 ; p=.000) \text {, la policía }(\beta=.567 ; p=.000) \text { y } \\
\text { los derechos humanos }(\beta=.324 ; p=.000) \text {. La atención a las noticias en } \\
\text { la radio incidió sobre la confianza en el sistema }(\beta=.078 ; p=.010) \text { y } \\
\text { los derechos humanos }(\beta=.112 ; p=.05) \text {. En el caso del seguimiento } \\
\text { de la delincuencia en la prensa, influyó negativamente en la confianza al } \\
\text { sistema }(\beta=-.091 ; p=.05), \text { la policía }(\beta=-.092 ; p=.05) \text { y los derechos } \\
\text { humanos }(\beta=-.129 ; p=.05)\end{array}$ \\
\hline
\end{tabular}


Tabla 1 (continuación). Estado del arte

\begin{tabular}{llll} 
Año & Autor & Muestra & Instrumentos \\
\hline \multirow{2}{*}{2010} & Oliva, & Residentes & Inventario \\
& Hernández \& & $(N=825)$ & estado Rasgo y \\
& Calleja & & $\begin{array}{l}\text { Expresión de Ira } \\
\text { (STAXI-2) }\end{array}$
\end{tabular}

Hallazgos

2010 Ruíz

2010 Sánchez \& Cerezo

2010 Silveira, Assunçào, Figeiredo \& Beato

2011 Álvarez, Núñez, Álvarez, Dobarro, Rodríguez \& González

2012 García
Residentes Escala de Efica$(N=400) \quad$ cia Colectiva, Escala de Cultura Ciudadana,Escala de Clima Emocional, Escala de Miedo Difuso al Delito, Escala de Satisfacción con la Policía, Escala de Miedo Concreto, Escala de Victimización

Estudiantes Test Bullying $(N=426)$

Estudiantes

Cuasi-experimento

Estudiantes Cuestionario de $(N=638) \quad$ Violencia Escolar Revisado

Estudiantes Escalas de per$(N=188)$
Los deseos de expresar ira correlacionaron con la expresión externa de ira $(.616 ; p<.05)$ y con el control externo de la ira $(-.271 ; p<.05)$. El sentimiento de ira con el temperamento iracundo $(.598 ; p<.05)$ y con el control externo de la ira $(-.301 ; p<.05)$. El temperamento iracundo con la expresión externa $(.618 ; p<.05)$ y con el control externo $(-.387$; $p<.05)$. La reacción de ira con la expresión externa $(.479 ; p<.05)$ y con el control externo $(-.142 ; p<.05)$. El control interno con la expresión externa $(-.209 ; p<.05)$. Por último, la expresión interna con la expresión externa (.412). Encontraron diferencias significativas entre hombres y mujeres con respecto al sentimiento de ira $(t=1.992 ; p<.05)$, el temperamento iracundo $(t=-2.112 ; p<.05)$ y el control interno $(t=-2.965$; $p<.05)$

El nivel socioeconómico correlacionó con el temor al delito (-.149), eficacia colectiva (.191), cultura ciudadana (.269), victimización (-.117), clima emocional (.274). Asimismo, la victimización se asoció con temor al delito (.201), eficacia colectiva (.258), cultura ciudadana (.223), satisfacción con la policía (-.136), clima emocional (.3999). Por su parte, el temor al delito se relacionó con eficacia colectiva (-.264), cultura ciudadana (-.315), satisfacción con la policía (-.242), con la victimización (.170) y el clima emocional (-.475). La eficacia colectiva se vinculó con cultura ciudadana (-554), satisfacción con la policía (.229) y el clima emocional (.382). La cultura ciudadana correlacionó con la satisfacción con la policía (.358), la victimización (-.142) y con el clima emocional (.567). La satisfacción con la policía se asoció con la victimización (.114) y el clima emocional (.333). Por último, la victimización con el clima emocional (-.295)

Encontraron diferencias entre agresores y victimas. Los primeros manifestaron necesidades económicas y sociales de integración escolar y los segundos necesidades afectivas parentales. A medida que se cumplen características de sexo, edad y escolaridad se incrementan las probabilidades de riesgo y vulnerabilidad en torno a la violencia bullying

El programa de prevención del delito implementado en una favela de violencia media resultó poco significativo en otras favelas más violentas de la ciudad de Belo Horizonte. El número de homicidios antes y después del programa no varió significativamente

Establecieron correlaciones positivas entre la violencia cotidiana y la violencia a través de las Tecnologías de Información y Comunicación. Encontraron diferencias significativas entre sexos y entre centro y periferia espacial

La percepción mediática del accionar gubernamental incidió positivamente sobre la percepción de la inseguridad pública $(\beta=.36 ; p<.001)$ nar policiaco, inseguridad pública, mediatizada del accionar policiaco y mediatizada del accionar gubernamental 
a la ansiedad, el enojo, la preocupación, el miedo y la desconfianza como factores de estrés referentes a experiencias de agresión, robo, secuestro, extorsión, asesinato o violación (Fernández, Revilla, \& Domínguez, 2010; Oliva, Hernández, \& Calleja, 2010), han establecido diferencias significativas entre hombres y mujeres así como entre los grupos a los que pertenecen o quieren pertenecer al haber presenciado o sufrido alguna experiencia delictiva (Bolívar, Contreras, Jiménez, \& Chaux, 2010; Elizalde, 2010; Sánchez \& Cerezo, 2010), han relacionado los contextos socioeconómicos y territoriales con el manejo de emociones (Gibson, Sullivan, Jones, \& Piquero, 2010) y sobre todo, han establecido una relación directa y positiva entre la confianza hacia las autoridades y la gobernanza local (Malone, 2010; Ruíz, 2010; Silveira, Assunçào, Figeiredo, \& Beato, 2010). Tales relaciones de experiencias cara a cara también fueron corroboradas en experiencias delictivas a través de tecnologías de información (Álvarez et al., 2011; García, 2011).

Sin embargo, los instrumentos para medir las emociones relativas a la seguridad pública han sido desarrollados considerándolos como evidencia de evaluación de políticas públicas o como un diagnóstico del estrés ciudadano ante la acción gubernamental. A partir de tales criterios, los instrumentos han ponderado directamente la opinión pública en torno al delito soslayando la cotidianidad en la que está inmersa la sociedad civil y que se deriva de las decisiones gubernamentales de procuración de justicia.

En este tenor, las experiencias cotidianas en el transporte, durante un paseo o una llamada telefónica estarían relacionados con acontecimientos de violencia tales como asaltos, secuestros, extorsiones, asesinatos o desapariciones que podrían disminuir o incrementar la confianza hacia los instrumentos de seguridad pública tales como policía judicial, ministerio público o tecnologías de vigilancia. En tales procesos, las emociones determinarían la evaluación de las autoridades a partir de activar experiencias directas o indirectas con la delincuencia. ¿Cuáles son las relaciones entre la ansiedad, la preocupación, el enojo y el miedo ante la acción gubernamental de prevención del delito o combate a la delincuencia?

Hipótesis. Las experiencias emocionales cotidianas de los ciudadanos están directamente relacionadas con sus evaluaciones de las autoridades en materia de prevención del delito o combate a la delincuencia (figura 1). En tal sentido, en un evento de riesgo e incertidumbre, la ansiedad estaría

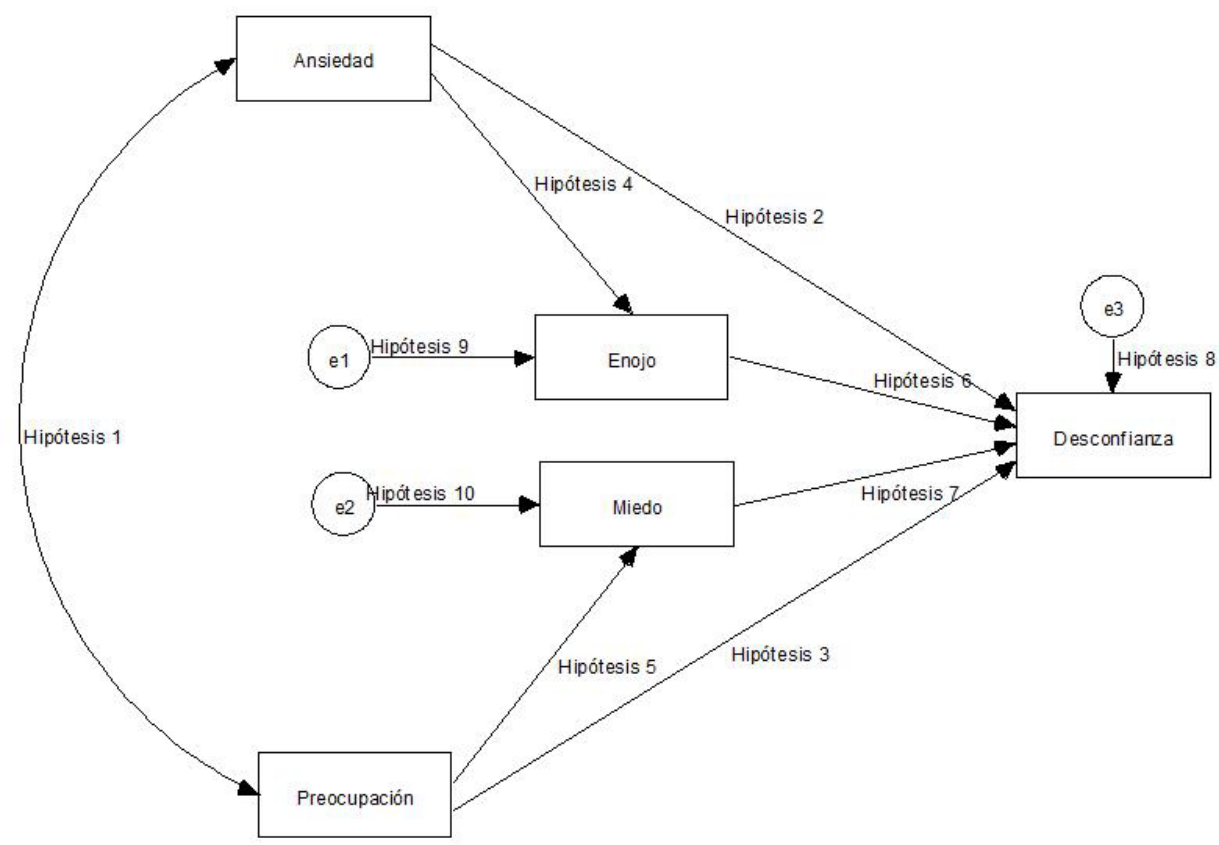

Figura 1. Trayectoria de relaciones entre emociones y evaluaciones 
Tabla 2. Normalidad, Confiabilidad y Validez de las Emociones

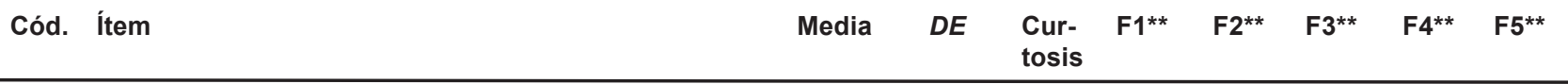

Inventario de ansiedad ( $0=$ nunca, $1=$ casi nunca, $2=$ a veces, $3=$ casi siempre, $4=$ siempre) alfa $=.68$

$\begin{array}{llllll}\text { R1 Al caminar volteo hacia atrás } & 1.14 & .41 & .141 & .368^{*} \\ \text { R2 } & \text { Al recibir llamadas escucho las mismas voces } & 1.28 & .26 & 1.52 & .161 \\ \text { R3 } & \text { Al salir de casa reviso cerraduras } & 1.02 & .37 & 1.62 & .162 \\ \text { R4 } & \text { Al subirme al transporte elijo los primeros asientos } & 1.71 & .41 & 1.73 & .674^{*} \\ \text { R5 } & \text { Al entrar a cualquier lugar oculto pertenencias } & 1.26 & .82 & 1.75 & .831^{*}\end{array}$

Inventario de preocupación ( $0=$ nada frecuente, $1=$ muy poco frecuente, $2=$ poco frecuente, $3=$ muy frecuente) alfa $=.61$

$\begin{array}{llllll}\text { R6 } & \text { Algún auto abandonado cerca de mi casa } & 1.54 & .79 & 1.66 & .821^{*} \\ \text { R7 } & \text { Alguna persona que silba por donde compro } & 1.16 & .81 & 1.57 & .130 \\ \text { R8 } & \text { Algún grupo que se reúne en mi calle } & 1.19 & .91 & 1.36 & .728^{*} \\ \text { R9 } & \text { Algún semáforo sin reparar por donde transito } & 1.01 & .95 & 1.42 & .128 \\ \text { R10 } & \text { Algún vendedor cerca del cajero automático que uso } & 1.81 & .71 & 1.74 & .729^{*}\end{array}$

Inventario de enojo $(0=$ ninguna vez, $1=$ muy pocas veces, $2=$ algunas veces, $3=$ muchas veces $)$ alfa $=.72$

$\begin{array}{llllll}\text { R11 } & \text { Al olvidar mis llaves } & 2.24 & .73 & 1.47 & .164 \\ \text { R12 } & \text { Al olvidar mi teléfono } & 2.15 & .82 & 1.42 & .317^{*} \\ \text { R13 } & \text { Al olvidar mi dinero } & 2.73 & .86 & 1.27 & .472^{*} \\ \text { R14 } & \text { Al olvidar mis compromisos } & 2.91 & .95 & 1.17 & .124 \\ \text { R15 } & \text { Al olvidar mis claves de acceso a Internet } & 2.01 & .97 & 1.90 & .361^{*}\end{array}$

Inventario de miedo $(0=$ nada, $1=$ muy poco, $2=$ poco, $3=$ mucho, $4=$ muchísimo $)$ alfa $=.76$

$\begin{array}{llllll}\text { R16 } & \text { Un asalto con arma de fuego } & 1.91 & .71 & 1.15 & .413^{*} \\ \text { R17 } & \text { Un secuestro exprés } & 1.82 & .58 & 1.17 & .412^{*} \\ \text { R18 } & \text { Una extorsión telefónica } & 1.47 & .47 & 1.68 & .326^{*} \\ \text { R19 } & \text { Un asesinato pasional } & 1.21 & .57 & 1.94 & .315^{*} \\ \text { R20 } & \text { Una desaparición forzada } & 1.65 & .35 & 1.96 & .467^{*}\end{array}$

Inventario de desconfianza ( 1 = totalmente en desacuerdo, $2=$ en desacuerdo, 3 de acuerdo, $4=$ totalmente de acuerdo, ) alfa $=.60$ catar a alguien se trata

$\begin{array}{lllll}\text { R22 Más vale ministerio público conocido que conocerlo ante } & 3.91 & .37 & 1.54 & .125\end{array}$ una denuncia

$\begin{array}{llllll}\text { R23 Las patrullas vigilan, pero los intereses de la delincuen- } & 3.05 & .92 & 1.48 & .467^{*}\end{array}$ cia organizada

$\begin{array}{lllllll}\text { R24 El tráfico es provocado por los agentes de tránsito } & 3.85 & .89 & 1.38 & .368^{*}\end{array}$

$\begin{array}{llllll}\text { R25 Mas vale instalar video-cámaras de seguridad que con- } & 3.61 & .97 & 1.69 & .316^{*}\end{array}$ tratar a un vigilante para prevenir el delito

Nota: Curtosis $=3.211$, Bootstrap $=.000 ; K M O=.762$, Bartlett $=.000 ;\left({ }^{* *}\right) \mathrm{F} 1=$ Ansiedad $(31 \%$ de varianza explicada),

$F 2$ = Preocupa-ción (25\% de varianza explicada), F3 = Enojo (19\% de varianza explicada), F4 = Miedo (12\% de varianza explicada), $F 5=$ Desconfianza ( $7 \%$ de varianza explicada). $\left(^{*}\right) P \geq 0.005$ 
relacionada con la preocupación (hipótesis 1) ya que un incremento en ésta última podría generar una desconfianza en las autoridades (hipótesis 2), empero una mayor preocupación por la seguridad personal estaría vinculada a un aumento en la confianza de combate a la delincuencia (hipótesis 3). Ahora bien, la ansiedad podría incrementar su efecto sobre la desconfianza hacia la acción gubernamental a través del enojo (hipótesis 4), aunque la preocupación también podría explicar la variabilidad de la desconfianza a través del miedo (hipótesis 5). Es decir, un mayor enojo propiciaría una mayor desconfianza (hipótesis 6), pero un excesivo miedo generaría una menor desconfianza porque la ciudadanía se sentiría indefensa ante la delincuencia y sus autoridades serían un refugio (hipótesis 7). No obstante, existen otras emociones, tales como la decepción, que estaría influyendo sobre la desconfianza (hipótesis 8). La asertividad o la venganza sobre el enojo (hipótesis 9) así como la autoestima y la depresión sobre el miedo (hipótesis 10).

\section{MÉTODO}

Diseño. Se llevó a cabo un estudio correlacional y transversal.

Muestra. 170 habitantes seleccionados itencionalmente de una comunidad huasteca con edad promedio de 23.6 años $(D E=9.3)$ e ingreso económico mensual de 670 dólares estadounidenses ( $D E=10.8$ USD).

Instrumento. Inventario de ansiedad (cinco opciones de respuesta que van desde "nunca"

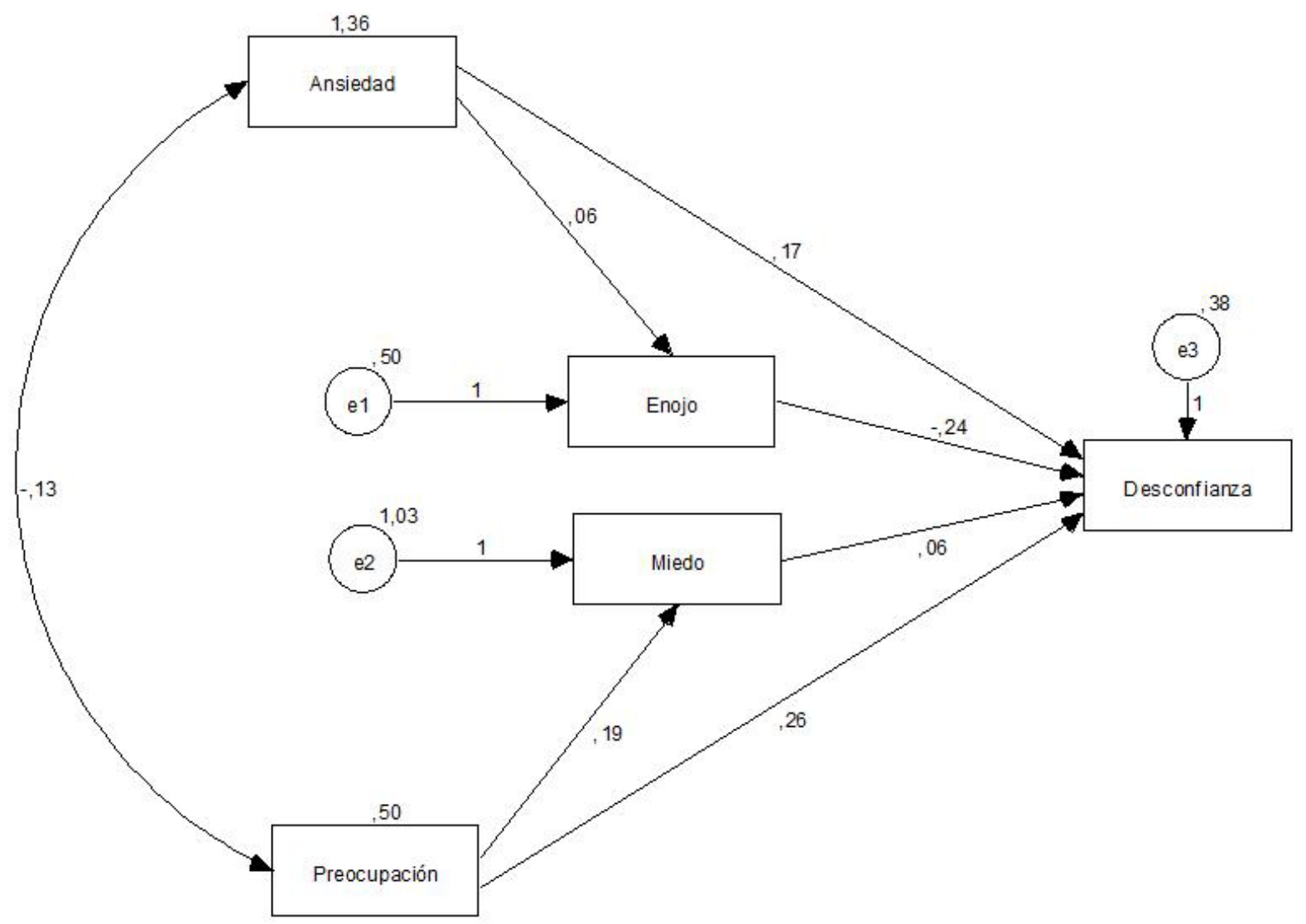

Figura 2. Estructura de relaciones entre emociones y evaluaciones 
Tabla 3. Covarianzas entre los factores

\begin{tabular}{llllllll}
\hline $\mathbf{X}^{2}$ & gl & $\mathbf{P}$ & RMR & GFI & AGFI & NFI & RMSEA \\
\hline $\mathbf{1 . 8 0 8}$ & 3 & .613 & .058 & .984 & .921 & .867 & .090 \\
\hline
\end{tabular}

Tabla 4. Parámetros de ajuste

\begin{tabular}{llllll}
\hline & Ansiedad & Preocupación & Enojo & Miedo & Desconfianza \\
\hline Ansiedad & 1.496 & & & & \\
Preocupación & $-.129^{*}$ & 1.360 & & & \\
Enojo & -.023 & .076 & 1.502 & 1.050 & 1.466 \\
Miedo & .093 & $-.236^{*}$ & -.074 & .060 & \\
Desconfianza & $.117^{*}$ & $.173^{*}$ & $-.115^{*}$ & \\
\hline
\end{tabular}

Nota: $\left({ }^{*}\right) p \geq 0.005$

hasta "siempre"), preocupación (cuatro opciones desde muy frecuente hasta nada frecuente), enojo (cuatro opciones desde muchas veces hasta ninguna vez, miedo (cinco opciones desde muchísimo hasta nada) y escala de desconfianza (cuatro opciones desde totalmente de acuerdo hasta "itotalmente en desacuerdo).

Análisis. Se estimó la normalidad con una curtosis multivariable inferior a 5 , una consistencia interna a partir de un alfa superior a .60, la adecuación a partir de un valor KMO superior a .50 , una significancia menor a .05 para esfericidad con la prueba de Bartlet y la validez se estableció con una correlación factor-item superior a .300 (tabla 1).

\section{RESULTADOS}

Una vez establecidas la normalidad, confiabilidad y validez, se estimaron las covarianzas entre los factores (tabla 3). Los valores cercanos a la unidad fueron considerados como evidencia de colinealidad mientras que los próximos a cero fueron entendidos como relaciones espurias.

La asociación negativa entre ansiedad y preocupación $(\Phi=-.129)$ denota una ambivalencia de emociones que se genera ante hechos cotidianos de inseguridad. En contraste, la relación positiva entre la ansiedad para con la desconfianza $(\Phi=$ .117) supone que un incremento en la ansiedad está vinculado con un aumento en la desconfian- za a las autoridades. Respecto a la preocupación, su correlación negativa con el miedo $(\Phi=-.236)$ alude a que la ciudadanía está se encuentra en un estado de ambivalencia ante la acción gubernamental en materia de prevención del delito o combate a la delincuencia. Sin embargo, el vinculo positivo de la preocupación con la desconfianza ( $\Phi$ $=.173$ ) advierte un estado de indefensión ya que la ciudadanía se preocupa cada vez cuando su confianza disminuye en quienes la gobiernan. Por ello, la asociación entre enojo y desconfianza resultó negativa $(\Phi=-.115)$ la cual sugiere que una mayor confianza está ligada con un menor enojo.

No obstante que las covarianzas especificaron algunas relaciones entre los factores emocionales, se calculó la estructura de las trayectorias supuestas (figura 2). Los resultados muestran que la desconfianza es explicada positivamente por la preocupación $(\beta=.26)$ y negativamente por el enojo $(\beta=-.24)$. Sin embargo, la incidencia de otros factores (la decepción entre otras emociones) no incluidos en el modelo inciden sobre la desconfianza $(\beta=.38)$.

Para contrastar cada una de la hipótesis, se calcularon los parámetros de dependencia, ajuste y residuales (tabla 4). Debido a que el primer estadístico $\left(X^{2}=1.0808 ; 3 \mathrm{gl} ; p=.613\right)$ es sensible al tamaño de la muestra, se estimó el índice de bondad de ajuste $(G F I=.984)$, índice de bondad de ajuste ajustado $(A G F I=.921)$, índice de ajuste normado $(N F I=.867)$ así como el residuo cuadrático medio $(R M R=.058)$ y el error de apro- 
ximación cuadrático medio $(R M S E A=.090)$. Tales valores se consideraron como evidencia de aceptación de todas las hipótesis con excepción de las hipótesis 4 y 7 ya que sus valores están cercanos a cero y fueron asumidos como relaciones espurias. Es decir, entre el la ansiedad y el enojo ( $\beta=$ .06) y entre el miedo y la desconfianza $(\beta=.06)$ hay otros factores (la asertividad y la venganza o la autoestima y la depresión entre muchos otros) que estarían influyendo dichas relaciones.

\section{DISCUSIÓN}

El presente estudio ha establecido un modelo de trayectorias de relaciones entre factores emocionales que explican la desconfianza hacia las autoridades en situaciones cotidianas de inseguridad. Sin embargo, otros factores emocionales (la decepción, entre otros) estarían influyendo sobre la confianza hacia la acción gubernamental en materia de prevención del delito o combate a la delincuencia.

En referencia al estudio de Oliva, Hernández y Calleja (2010) en el que encontraron asociaciones entre el enojo dirigido a un grupo y el temperamento, la presente investigación encontró una relación negativa entre el enojo y la desconfianza hacia las autoridades. A medida que las personas incrementan su molestia consigo mismos, aumentan su confianza hacia sus autoridades.

Respecto al trabajo de Malone (2010) en el que la confianza hacia las autoridades está en función del miedo a la delincuencia, en el presente estudio se encontró que entre el miedo al secuestro, asalto, desaparición, violación o asesinato y la desconfianza hacia la acción policiaca otras variables inciden esta relación.

Ambos estudios relativos al enojo y al miedo como determinantes de la confianza hacia la acción gubernamental deberán ser complementados con otros factores. En este sentido, García-Lirios (2012) estableció el efecto de la percepción de inseguridad a través de los medios de comunicación. Es decir, las experiencias de inseguridad tendrían un componente perceptual derivado de observar los hechos de violencia en los medios de comunicación.

\section{REFERENCIAS}

Álvarez, D., Núñez, J., Álvarez, L., Dobarro, A., Rodríguez, C., \& González, P. (2011). Violencia a través de las tecnologías de la información y la comunicación en estudiantes de secundaria. Anales de Psicología, 27, 221-231.

Bolívar, C., Contreras, J. Jiménez, M., \& Chaux, E. (2010). Desentendimiento moral y dinámicas del robo escolar. Revista de Criminología, 52, 243-261.

Elizalde, A. (2010). Estudio descriptivo de las estrategias de afrontamiento del bullying en profesorado mexicano. Education \& Psychology, 8, 353-372.

Fernández, C., Revilla, J., \& Domínguez, R. (2010). Emotions elicited by television violence. Scientific Journal of Med ia Literacy, 36, 97-104.

García-Lirios, C. (2012). Estructura perceptiva de inseguridad en universitarios. Liberabit, 17, 1-10.

Gibson, C., Sullivan, C. Jones, S., \& Piquero, A. (2010). Does it take village? Assessing neighborhood influences of children's self-control. Journal of Research in Crime and Delinquency, 47, 31-62.

Malone, M. (2010). The verdict is in: the impact of crime on public trust in Central American Justice System. Journal of Politics in Latin America, 3, 99-128.

Oliva, F., Hernández, M., \& Calleja, N. (2010). Validación de la versión mexicana del inventario de expresión de ira estado rasgo (STAXI-2). Acta Colombiana de Psicología, 13, 107-117.

Ruíz, J. (2010). Eficacia colectiva, cultura ciudadana y victimización: un análisis exploratorio con sus relaciones con diversas medidas de miedo al crimen. Acta Colombiana de Psicología, 13, 103-114.

Sánchez, C., \& Cerezo, F. (2010). Variables personales y sociales relacionadas con la dinámica bullying en escolares de educación primaria. Journal of Research in Education Psychology, 8, 1015-1032.

Silveira, A., Assunçào, R., Figeiredo, B., \& Beato, C. (2010). Impact of the staying alive programo on the reduction of homicides in a community in Belo Horizonte. Revista Saùde Pùblica, 44, 1-6. 